\title{
Inkjet-printed paper-based surface enhanced Raman scattering (SERS) sensors for the detection of narcotics
}

\author{
Li-Lin Tay ${ }^{1}$ (i) $\cdot$ Shawn Poirier ${ }^{1}$ Ali Ghaemi ${ }^{1} \cdot$ John Hulse ${ }^{1}$
}

Received: 3 January 2022 / Accepted: 23 February 2022 / Published online: 7 March 2022

(c) Crown 2022

\begin{abstract}
Recent advances in inkjet-printing of advanced materials have provided a versatile platform for the rapid development and prototyping of sensor devices. We have recently demonstrated inkjet-printed surface enhanced Raman scattering (SERS) sensors on flexible substrates for the detection of variety of small molecules [Tay et al. in Front Chem 9:680556 (2021); Tay et al. in J Raman Spectrosc 52:563 (2020)]. These flexible SERS sensors have many advantages for performing point-ofsampling testing, among them liquid or aerosol filtration and swabbing capabilities. These simple sampling and separation features make these inkjet-printed paper-based sensors ideal for field applications. SERS detection of molecules with poor binding affinity towards the plasmonic surfaces of the sensors tends to be inefficient. A surface functionalization approach has been applied to SERS sensors to improve the molecule affinity and hence their detection sensitivity. In this paper, we investigate the optimization of SERS sensor fabrication to achieve optimal performance. Three performance criteria: diffuse reflectance, SERS background intensity from the as-printed blank sensors and SERS performance of sensors exposed to the benzenethiol reporter molecule, are characterized carefully to derive the optimal inkjet-printing conditions for producing the best performing SERS sensors. Additionally, we demonstrate the use of a simple potassium iodide functionalization scheme to improve the detection sensitivity for narcotics such as fentanyl by two orders of magnitude.
\end{abstract}

\section{Introduction}

Fentanyl is a potent synthetic opioid originally developed for pain relief and management of severe pain such as, for example, in cancer patients. It has a potency that is $80-100$ times that of morphine. Consumed as a drug outside of medical treatment, users experience a rush of euphoria, sedation and respiratory depression [1]. It is often mixed with other street drugs to increase their potency and increasingly it has become the choice of drug by users. The drug overdose crisis due to the use of fentanyl and its analogue is having devastating impacts on communities and families across Canada. Safe drug consumption sites in many of the Canadian cities provide users with a number of drug checking options to allow the users to make an informed choice [2]. Analytical methods such as fentanyl test strips, GC-MS, FTIR and Raman spectroscopy are among the common analytical methods employed for drug checking at the safe

Li-Lin Tay

lilin.tay@nrc-cnrc.gc.ca

1 Metrology Research Centre, National Research Council Canada, Ottawa, ON K1A0R6, Canada consumption sites in Canada [3]. Advances in miniaturization of photonic components have enabled the development of many affordable hand-held Raman analyzers. Raman spectroscopy is a non-invasive technique for chemical and biomolecular identifications $[4,5]$. Unfortunately, the technique is limited by the small scattering cross-sections of molecules which compromises the sensitivity of detection. This inherent limitation can be overcome by using surface enhanced Raman scattering (SERS) substrates [6] to improve the detection sensitivity and this has been demonstrated over a broad range of applications from security [7, 8] to food safety $[9,10]$. The extraordinary sensitivity of SERS arise from the resonant excitation of coupled plasmonic nanostructures. Coupling of plasmonic nanostructure allows an electromagnetic field to be focused and localized within the junction hot-sites between the nanostructures components (known as the SERS "hot-sites") [11]. It is important to note that the strength of this enhancement is highly dependent on the geometrical arrangement, morphology and degree of coupling of the nanostructures in the optical near-field [12, 13]. Another factor that plays a critical role in overall SERS performance is the ability of the molecule to interact with the SERS hot-site. Colloidal Ag or Au nanoparticle are among 
some of the most popular SERS substrates used for a broad range of bio-sensing and chemical sensing applications [14, 15]. In this work, we take advantage of the well-studied and understood colloidal nanoparticles and integrate them onto a flexible paper substrate for versatile SERS applications.

Recent advances in inkjet-printing of advanced materials have provided a versatile platform for the rapid development and prototyping of sensor devices. We have recently demonstrated inkjet-printed surface enhanced Raman scattering (SERS) sensors on flexible substrates for the detection of small chemical molecules [16, 17]. These flexible SERS sensors have good batch-to-batch uniformity and many advantages for performing point-of-sampling testing such as liquid or aerosol filtration and swabbing capabilities. These simple sampling and separation properties make these inkjet-printed paper-based sensors ideal for field applications. SERS exploits the unique vibrational signature of an analyte molecule and in practice, it is often coupled with spectral recognition software for the identification of molecules of interest. Most SERS sensors are fabricated as nonfunctionalized plasmonic nanostructures in a single device. Because SERS is an optical near-field effect, it works best with molecules that have good binding affinity towards the plasmonic surfaces (such as thiols). SERS detection of molecules with poor binding affinity towards the plasmonic surfaces (such as cannabinoids) tends to perform poorly. This can be improved by exploiting surface functionalization to help sequester or attract the molecules of interest to the plasmonic surfaces [18]. The functionalization concept has been applied in SERS detection for a number of years. However, its applications have been mostly limited to optimization using a particular functional group to target one particular chemical analyte, for example, the use of alkyl thiol to improve glucose SERS sensing [19]. Once the surface has been functionalized and tailored for one specific analyte, it can often render the entire surface in-accessible or insensitive to other molecules of interest. For example, plasmonic surfaces functionalized by alkyl thiol to improve the detection of molecules with poor binding affinity such as glucose [19] are usually no longer active for detection of other molecular species. A better trade-off is to introduce halide ions such as $\mathrm{Cl}$ or I ions, which displace weakly adsorbed carbonaceous species from the plasmonic surfaces while allowing a broad spectrum of chemicals of interest to continue to interact with the plasmonic surfaces. This will permit the SERS sensors to detect a broader range of chemicals rather than tailoring the surface to target just one single species. In this paper, we will demonstrate the optimization of functionalized plasmonic ink formulations, inkjet-printing conditions and improvement in SERS sensitivity through iodide treatment of the printed sensors. We will discuss the performance of differently prepared SERS devices for the detection of narcotics.

\section{Experimental}

\section{Inkjet-printing of SERS sensors}

Colloidal Au nanoparticles (AuNP) with nominal size of $50 \mathrm{~nm}$ was purchased from BBI Solutions. The as-purchased $\mathrm{Au}$ sol was then cleaned and concentrated by centrifugation to remove excess citrate anion and obtain AuNP ink formulation suitable for inkjet-printing. Following centrifugation, the supernatant was decanted and the pellet re-suspended to $1 / 4$ of the original volume with deionized water. This produces an ink formulation that is four times the concentration of the as-purchased Au sol and removes as much as possible of the excess citrate ion from the initial colloidal sol. Figure 1a shows a photograph of the four-time concentrated colloidal Au sol (labelled as $4 \times$, Fig. 1a) and the aspurchased colloidal sol (labelled as $1 \times$, Fig. 1a). The image in Fig. 1a shows that the 4X concentrated Au sol is well dispersed in the aqueous solution and free from signs of formation of large aggregates. It is important to work with a stable colloidal sol as large aggregates can potentially clog up the printing nozzle. The concentration of the fourtime\&nbsp; concentrated AuNP ink is $\sim 2.25 \times 10^{11} \mathrm{NP} / \mathrm{mL}$. We have modified a commercial Epson piezoelectric inkjet printer (Epson WF-7110) to carry out the sensor printing. In a typical printing run, approximately $3-6 \mathrm{~mL}$ of AuNP ink are prepared for the printing of a full batch of the sensors. Printing of the sensors uses only a fraction of the loaded ink, most of the NP ink are lost in the blotting and initialization process when the printer draws a significant amount of ink through the printing head to ensure normal jetting condition is achieved. Details of the printing and parameter optimization are can be found in reference [16]. All results discussed in this proceeding manuscript are obtained from sensors printed with the Epson inkjet printers. The inkjet printer has a printhead with 800 (two lines of 400) micromachined jetting nozzles. Ink drop size ranges from 32 to $1.5 \mathrm{pL}$ with a printing resolution of $5760 \times 1440 \mathrm{dpi}$. In a single printing pass, the amount ink that can be deposited ranges from 19 to $420 \mathrm{~nL}$ which translates into approximately 4 to $94 \times 10^{6}$ NPs deposited onto the sensor in each printing pass. The precise amount of NPs deposited in each printing pass cannot be estimated better than the range provided here due to the limitation in control of the pulse that drives the piezoelectric element and the pre-programmed printing setup of the commercial hardware and software. However, the above number does provide a general range in the amount of NPs deposited each time when the sensors are printed. There is a limited amount of NP ink that can be deposited in a single printing step. In order to optimize the performance of the SERS sensors, multiple printing passes are required to ensure higher loading of AuNPs on the paper substrates. 
Fig. 1 a Colloidal NPs used for the printing of SER sensors.

The two vials labelled $4 \mathrm{X}$ are four times more concentrated NP ink used for printing of sensors. The vial labelled as $1 \times$ is the as-purchased colloidal Au sol. b Scanning electron microscopy image of AuNPs printed on Whatman 44 filter paper. $\mathbf{c}$ Two photographs of test sensors printed with 1-10 printing passes. The number below each pad indicates the number of passes. $\mathbf{d}$ A typical array of printed SERS sensors printed with 6 printing passes
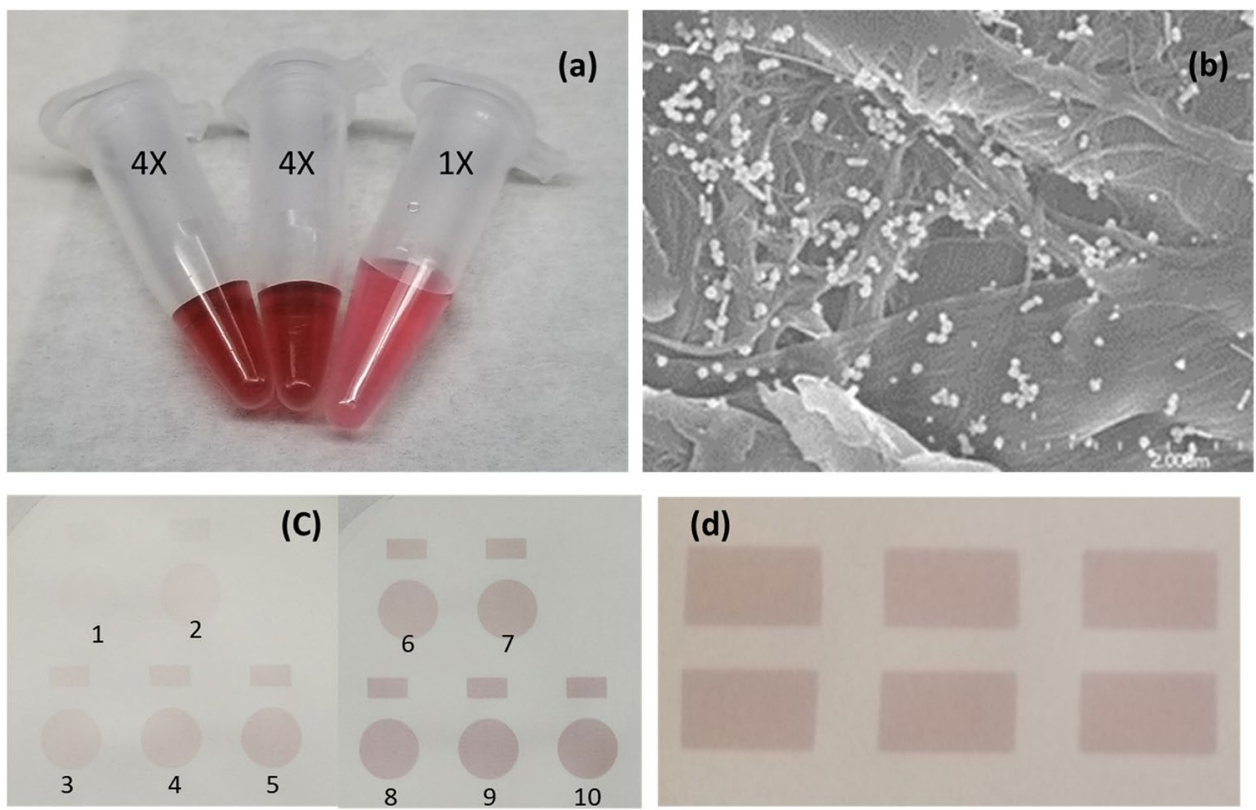

All sensors are printed on Whatman 44 filter paper purchased from Sigma-Aldrich and used as is. The filter paper used for this work has a thickness of $200 \mu \mathrm{m}$ and pore diameter of $3 \mu \mathrm{m}$. We did perform printing of sensors with other types of chromatography papers but found the best results were obtained from Whatman 44 substrates. For example, sensors were also test-printed on Whatman grade 1 chromatography (Chroma1) filter paper. We have noticed that sensors printed on the chromatography grade paper produce extremely high background counts as compared to the sensors printed on the Whatman 44 substrate. This is likely due to the residual chemicals that were used to treat the chromatography paper which are then solvated by the AuNP ink and manifested in the increase of the carbonaceous background observed for the blank sensors.

Fentanyl analytical standards were purchased from Sigma-Aldrich as a $1 \mathrm{mg} / \mathrm{mL}$ methanol solution. The fentanyl solution is further diluted with deionized water to lower concentrations for SERS sensor exposure.

\section{Optical characterization and electron microscopy}

As these sensors were designed for field applications, all Raman spectra presented here were acquired using a hand-held spectrometer, "ReporteR" from SciAps. The "ReporteR" is equipped with $70 \mathrm{~mW}, 785 \mathrm{~nm}$ excitation laser; a 2048 pixel TE cooled CCD array detector and an 1800 lines $/ \mathrm{mm}$ grating. The instrument covers a spectral range of $300-2500 \mathrm{~cm}^{-1}$ with $12 \mathrm{~cm}^{-1}$ spectral resolution. With the right angle attachment, the laser illumination spot size is approximately $25 \mu \mathrm{m}$. The system is connected to a laptop for spectral acquisition and manipulation for all the measurements performed in this study. The spectrometer was controlled by software, "Nuspec", supplied by the manufacturer. Before each experiment, the system was validated and calibrated using the manufacturer supplied polystyrene reference standard and the manufacturer recommended procedure.

The same sensors were also characterized using a commercial microRaman spectrometer, Horiba LabRAM HR. The system is equipped with a motorized XY scanning stage. In order to match the optical characterization condition similar to that of field instrumentation, the printed samples are sampled under focused $785 \mathrm{~nm}$ laser irradiation. The laser was focused through a 10X objective with an NA of 0.25 .

Diffuse reflectance spectra were acquired with the diffuse reflectance accessory of a Perkin Elmer Lambda 360 spectrophotometer. This is a double beam instrument. The reference beam illuminates a diffuse reflectance PTFE reflectance standard provided by the manufacturer.

Printed sensors were characterized by a field emission scanning electron microscope (Hitachi SU5000 SEM) operating at $10.0 \mathrm{kV}$.

\section{Results and discussions}

A paper-based SERS substrate has excellent flexibility and is quite forgiving in its handling. A typical array of inkjetprinted SERS sensor is shown Fig. 1c and d. The pink active area with the AuNP deposit is clearly visible due to the resonant scattering of the large AuNPs. Figure 1b shows a scanning electron microscopy (SEM) image of a printed sensors with six printing passes. The bright-coloured NPs randomly 
decorate the cellulose fibre. Although there are some NPs that appeared to be single particles, the majority of the NPs on the image have formed into small aggregates. This is extremely important as the majority of the SERS signals are generated from aggregated AuNPs which sustains strong field enhancements upon resonant optical excitation. The random distribution of the AuNP aggregate on the paper substrate will result in spot-to-spot variation of SERS signal. In this study, all of the Raman measurement are acquired with the hand-held Raman analyzer with the excitation laser focused to $\sim 25 \mu \mathrm{m}$ diameter. This large beam spot will cover many of the aggregates and to some degree average out the SERS signals from the randomly distributed AuNP aggregates. We have also examined the use of the receiver operating characteristic (ROC) method to better understand the binary quantification of the inkjet-printed SERS sensors [20].

An array of test sensors which have been printed as rectangular $(0.5 \mathrm{~cm} \times 1 \mathrm{~cm})$ and circular $(1 \mathrm{~cm}$ diameter $)$ pairs is shown in Fig. 1c. The number directly below each printed pair shows the number of printing passes the sensors has been prepared with. The colour from a single print pass is quite weak and is nearly invisible in Fig. 1c. As the number of printing passes increase, the colour of the active area becomes increasingly redder/darker as the AuNPs loading on the paper substrate increases. The colour of the backside of the printed area remain unchanged indicating that most of the AuNPs are retained near the upper surface of the filter paper. The rectangular and circular-shaped active area pairs were printed for different tests. The rectangular sensor was used for SERS characterization in Fig. 2, whereas the circular sensor was used for diffuse reflectance study in Fig. 3. The circular shape is printed to match the measurement port of the diffuse reflectance accessory in the spectrophotometer. Once the printing is completed, the sensors can be simply cut up with scissors to prepare the samples for the various different measurements. This demonstrates the versatility of the paper-based sensors and its ease of batched production and subsequent sample preparation.

From SEM studies, we do observe that the AuNPs are randomly deposited on the cellulose fibre and some of the AuNP are deposited beyond the top most layer of the cellulose fibre. It is also evident that as the number of printing passes increase so does the size of the NP aggregates. This is observed in both SEM images as well as the background SERS spectra (Fig. 2a). In Fig. 2a, SERS background remain relatively low ( $\sim 5000$ counts) in the first two printing passes. SERS background showed very significant increase from 4 to 8 printing passes with a background of approximately $10 \mathrm{~K}, 15 \mathrm{~K}$ and $22 \mathrm{~K}$ counts for the sensors with 4, 6 and 8 printing passes. This is due to the formation of increasingly larger AuNP aggregates as the number of printing passes increases. The grey trace in Fig. 2a shows the Raman spectrum of the cellulose fibre which made up the filter paper. Interestingly, as the number of printing passes increase, the cellulose Raman bands become much more intense and they also appear to be broadened. This is particularly evident for the 880 and $1100 \mathrm{~cm}^{-1}$ bands. This is due to multiple scattering of the Raman emission in the presence of the heavily loaded AuNPs on the cellulose fibre particularly at the higher printing passes. Interestingly, the SERS background is reduced significantly if the sensors are measured when saturated with water. This is likely due to the partial index matching of the water and cellulose matrix which greatly reduces the scattering of the light and resulting in a significant reduction of the background.

In order to determine the number of printing passes that are optimal for SERS detection, sensors with different printing passes were exposed to $1 \mu \mathrm{M}$ of the Raman reporter molecule, benzenethiol (BT) for $30 \mathrm{~min}$. Figure $2 \mathrm{~b}$ shows the
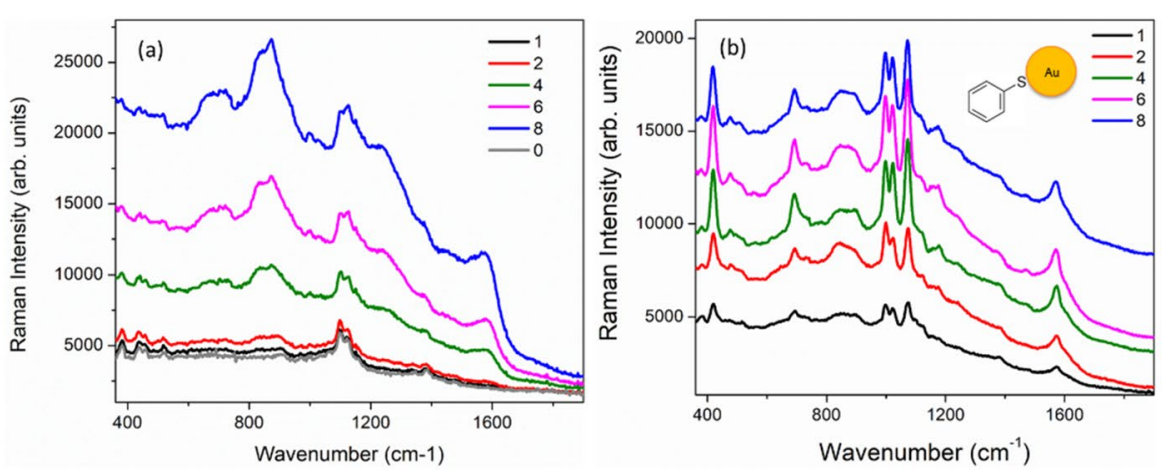

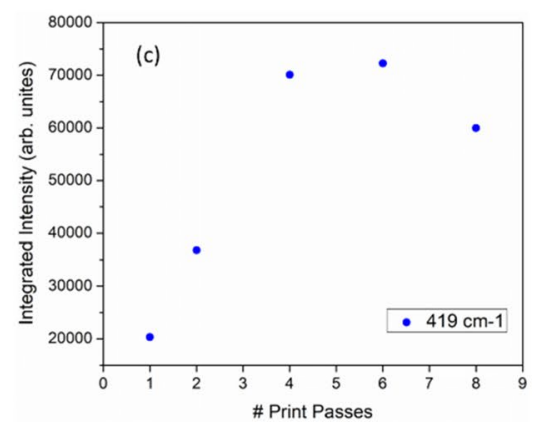

Fig. 2 Average SERS spectra of printed sensors. Each spectrum shown here is the average of 10 spectra acquired from 10 different spots of an individual sensor. a Background SERS spectra of the as-printed sensors after 1, 2, 4, 6, 8 printing passes. The grey trace labelled as 0 depicts the Raman spectrum of the paper substrate alone. All spectra were acquired with $5 \mathrm{~s}$ accumulation time. b
SERS spectra of the sensors prepared with different printing passes and exposed to $1 \mu \mathrm{M}$ of benzenethiol for $30 \mathrm{~min}$. All spectra were acquired with $2 \mathrm{~s}$ accumulation time. c Integrated intensity of the $419 \mathrm{~cm}^{-1}$ Raman vibrational band of the BT SERS spectrum from the measured sensors in (b) 

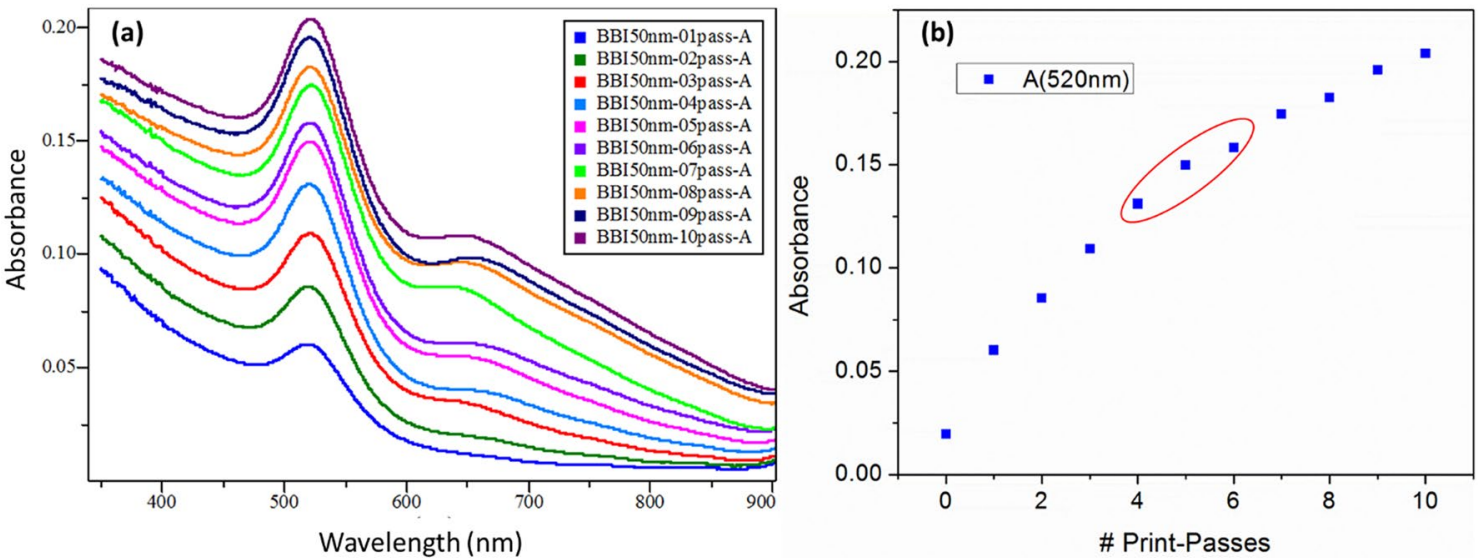

Fig. 3 a Diffuse reflectance spectra of SERS sensors prepared with 1-10 printing passes. Each spectrum is the average of 5 spectra taken from different spots of an individual sensor. b Plot of the maximum absorbance of the $520 \mathrm{~nm}$ localized surface plasmon resonance band

SERS spectra of BT from the sensors with different printing passes. As the number of printing passes increases, so does the SERS intensity of BT. However, careful comparison of the SERS spectra for 6- and 8 printing passes (PP) shows that the SERS intensity of the 8-PP sensor is lower than that of the sensor with 6 printing pass. The result is even more striking for the sensor with 10 printing passes which showed an even lower BT signal intensity than did the 6 printing pass sensor. The printing pass studies indicate that optimal SERS performance is reached between 4-6 printing passes for the $50 \mathrm{~nm}$ AuNP ink formulation. Six printing pass sensors were therefore prepared for the subsequent studies of narcotics detection. It is worth noting that although the SERS performances is generally better with slightly larger aggregates, there is a trade-off that is worth considering. As the number of printing passes increase beyond a certain threshold, (in this case, 6 printing passes), SERS performance appears to taper off and eventually drop significantly at higher numbers of printing passes. This is due to the formation of larger and larger aggregates on the filter paper as the number of printing passes increases. It is understood that SERS hotsites are confined to the junction between coupled nanoparticles. However, the intensity of the local field is highly dependent upon the geometry, morphology and size of the nanoaggregates. We have performed electromagnetic simulations and correlated these with SERS intensity, structural morphology obtained from aggregates of varying sizes [12]. In ours and other studies $[12,21]$ it was found that as the number of nanoparticles in an aggregate increases beyond a certain threshold, the observed SERS intensity tapers off and eventually shows a marked decrease when the nanoparticle aggregate sizes increases beyond this threshold. This is the primary reason for the drop off in the SERS performance for a higher number of printing passes. as a function of the number of printing passes. Note the increase in $520 \mathrm{~nm}$ absorbance as the number of printing passes increases. The circled points in $\mathbf{b}$ shows the optimal (4-6) printing passes based on the SERS-BT study

Furthermore, we noted that there is a very significant jump in the background signal of the blank SERS sensors as seen in Fig. 2a. While the very large background signal indicates a higher level of SERS enhancement, it also makes the detection of an analyte of interest, particularly those with weak or no binding affinity towards the AuNP surfaces, much more challenging. Sensors with high background counts tend to perform very well for molecules with good binding affinity, as shown in the case of BT in Fig. 2b. However, weakly binding or non-binding molecules apparently cannot displace the surface adsorbed citrates or other carbonaceous molecules form the AuNP keeping the analyte from benefiting from the near-field enhancement of the SERS aggregates. Trying to discern the presence of a small peak from a weakly or non-binding molecular target against a very large background is much more challenging than from a sensor with smaller background. We have determined the optimal printing passes for the general operation of our sensors to be between 4 and 6 printing passes when prepared with the printing condition described in the experimental section. This is an empirical determination and that needs to be performed for each different ink formulation and printing condition.

To better monitor and control the amount of AuNP deposited on the paper substrate, measurement of the localized surface plasmon resonance (LSPR) is a good indicator of the loading of AuNPs on the paper substrate. The LSPR of colloidal AuNPs and plasmonic nanostructures is typically measured using a standard transmission or specular reflection set up of spectrophotometer. In the case of paper-based SERS sensor, AuNPs are deposited on a highly diffusively scattering filter paper substrate. A transmission or reflection measurement that collects only specular transmitted or reflected light will miss all but a very limited and small 
No functionalization

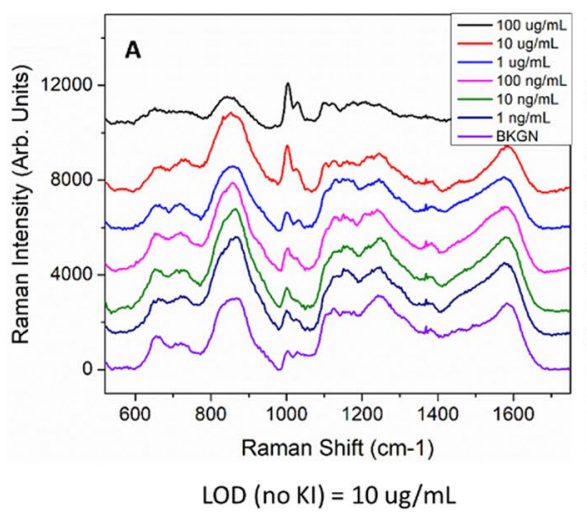

1- functionalization
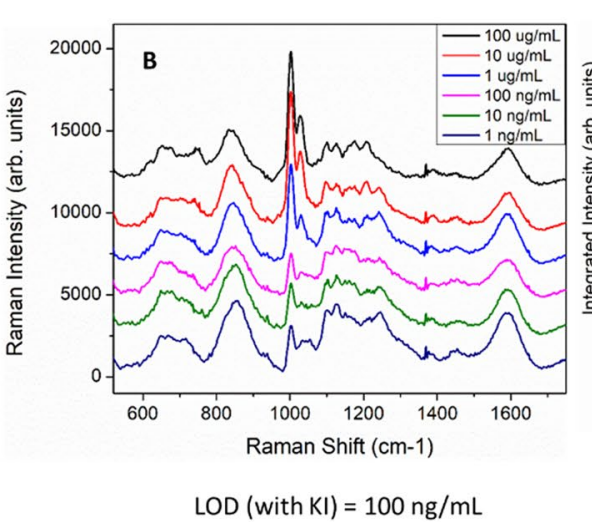

$\operatorname{LOD}($ with $\mathrm{KI})=100 \mathrm{ng} / \mathrm{mL}$

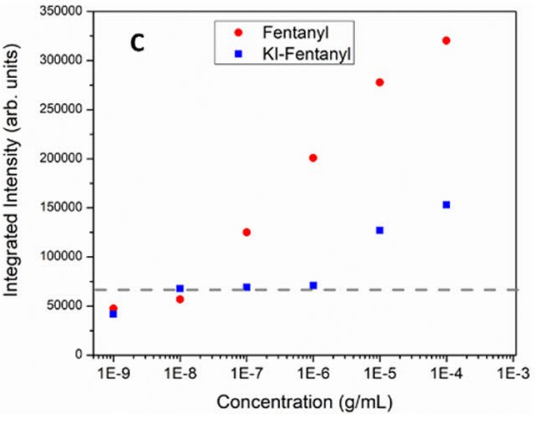

Each of the spectra shown here is an average of 10 spectra acquired from 10 different spots on the printed SERS sensor. c Integrated Raman intensity of the $1000 \mathrm{~cm}^{-1}$ band of the SERS spectra for the sensors exposed to various concentrations of fentanyl. The dashed line indicates the integrated intensity of the background spectra from the blank sensors fraction of the light scattered by the paper substrate. A more appropriate approach is to measure the diffuse reflectance from the substrate with the use of an integrating sphere. This ensures that all of the diffusively reflected light from the AuNP decorated paper substrate will be collected by the detector through use of integrating sphere accessory. Figure 3 a shows the diffuse reflectance spectra collected from the round sensors from Fig. 1c. Not surprisingly, as the number of printing passes increases, we see a monotonic increase of the $520 \mathrm{~nm}$ absorption band of the AuNPs which is indicative of the increase of AuNP loading on the filter paper (Fig. 3b). Also worth noting is a second absorption band which is further red-shifted and centred at $650-670 \mathrm{~nm}$, from the LSPR mode due to the formation of larger AuNP aggregates. This band is not visible in the first few printing passes but becomes quite noticeable starting at the 3rd printing passes. This mode also shows a slight shift as the number of printing passes increases. To further understand the first red- and then blue-shift of this LSPR mode, we will need further SEM and electromagnetic simulation modelling to determine the source of such shift. This work is currently underway. Figure $3 \mathrm{~b}$ shows the monotonic increase of the $520 \mathrm{~nm}$ absorption band as the number of printing passes increases. It is interesting to note that although the amount of AuNP ink deposited in each printing passes is expected to be roughly the same, the LSPR band does not increase linearly as seen in Fig. 3b. Rather the increase of the $520 \mathrm{~nm}$ absorption band seemed to follow more of a square-root function. It is important to note that all of the SERS measurement carried out in this work were excited with a $785 \mathrm{~nm}$ laser. We are actually probing the optical response within the 800-900 nm spectral window in Fig. 3a. The red circle in
Fig. $3 b$ shows the optimal printing passes that are typically used for the fabrication of our sensors.

Fentanyl is a powerful narcotic that has been the central focus of the opioid crisis [1]. Accidental overdose rates for users of fentanyl and its analogues has seen a dramatic rise, particularly during the pandemic period. Although the fentanyl molecule does not have an apparent binding moiety towards the AuNP surface, it does show a weak binding affinity potentially through the carbonyl or amine moiety. This allows for a reasonable expectation of successful SERS detection of the fentanyl molecule providing the first responders with additional sensitivity using a SERS detection scheme [16]. Figure 4a shows the response of our inkjet-printed SERS sensors exposed to fentanyl solutions. At higher concentrations (e.g. $100 \mu \mathrm{g} / \mathrm{mL}$ ), the fentanyl band at $1000 \mathrm{~cm}^{-1}$ can be clearly seen. Unfortunately, this band also overlaps with a native background band that exists on the blank SERS sensor and this compromises our ability to conclusively determine a positive detection event. Treating the as-printed SERS sensors with $1 \mathrm{mM}$ of KI aqueous solution significantly improves the signal strength of the $1000 \mathrm{~cm}^{-1}$ fentanyl vibrational band. Figure $4 \mathrm{~b}$ shows KI functionalized SERS sensors exposed to the same set of fentanyl solution as shown in Fig. 4a. The sensors pre-functionalized with iodide ion (through KI treatment) showed noticeable improvement. The fentanyl vibrational band from the iodide-functionalized sensors are easily doubled as compared to the un-treated sensors. Figure $4 \mathrm{c}$ shows the integrated intensity of the $1000 \mathrm{~cm}^{-1}$ band of the fentanyl SERS spectra for the un-functionalized sensors (blue square) and iodide-functionalized sensors (red circle). The dashed grey line indicates the integrated intensity of the background spectra from the blank sensors. The 
detection limit of the sensor is determined as the lowest concentration with an integrated intensity (of $1000 \mathrm{~cm}^{-1}$ band) that is clearly above the background level (grey dash line) as shown in Fig. 4c. For the un-functionalized sensors, the detection limit for fentanyl is $10 \mu \mathrm{g} / \mathrm{mL}$. Overall, the detection limit is improved by two orders of magnitude with the iodide treatment, reaching $100 \mathrm{ng} / \mathrm{mL}$.

\section{Conclusion}

In conclusion, we have demonstrated the fabrication of inkjet-printed SERS sensors with a commercially available Epson inkjet printer. We have carried out detailed characterizations of the diffuse reflectance measurements, SERS background from the as-printed blank sensors and SERS measurements from sensors exposed to BT reporter molecules on sensors that were fabricated with different printing passes. These studies allows us to determine empirically the optimal printing condition for best performing SERS sensors. We have also demonstrated the use of a simple halide functionalization to improve the SERS detection limit of fentanyl molecules by two orders of magnitude.

Funding Open access funding provided by National Research Council Canada.

Data availability The data that support the plots within this paper and findings of this study are available from the corresponding author on reasonable request.

\section{Declarations}

Conflict of interest The authors declare no competing interests.

Open Access This article is licensed under a Creative Commons Attribution 4.0 International License, which permits use, sharing, adaptation, distribution and reproduction in any medium or format, as long as you give appropriate credit to the original author(s) and the source, provide a link to the Creative Commons licence, and indicate if changes were made. The images or other third party material in this article are included in the article's Creative Commons licence, unless indicated otherwise in a credit line to the material. If material is not included in the article's Creative Commons licence and your intended use is not permitted by statutory regulation or exceeds the permitted use, you will need to obtain permission directly from the copyright holder. To view a copy of this licence, visit http://creativecommons.org/licenses/by/4.0/.

\section{References}

1. L.-L. Tay, S. Poirier, A. Ghaemi, J. Hulse, S. Wang, Paper-based surface enhanced Raman Spectroscopy sensors for field applications. J. Raman Spectrosc. 52, 563 (2020). https://doi.org/10.1002/jrs.6017

2. L.-L. Tay, S. Poirier, A. Ghaemi, J. Hulse, S. Wang, Iodide Functionalized Paper-Based SERS Sensors for Improved Detection of Narcotics. Front. Chem. 9, 680556 (2021). https://doi.org/10. 3389/fchem.2021.680556
3. R.A. Rudd, N. Aleshire, J.E. Zibbell, M. Gladden, Increases in drug and opioid overdose deaths. MMWR Morb. Mortal. Wkly Rep 64, 1378 (2016)

4. https://www.canada.ca/en/health-canada/services/substance-use/ supervised-consumption-sites.html

5. B. Wallace, T. van Roode, F. Pagan et al., What is needed for implementing drug checking services in the context of the overdose crisis? A qualitative study to explore perspectives of potential service users. Harm Reduct J 17, 29 (2020). https://doi.org/ 10.1186/s12954-020-00373-4

6. L.-L. Tay, R.G. Tremblay, J. Hulse, B. Zurakowski, M. Thompson, M. Bani-Yaghoub, Detection of acute brain injury by Raman spectral signature. Analyst 136, 1620 (2011)

7. O.J.R. Clarke, B.L. Goodall, H.P. Hui, N. Vats, C. Brosseau, Anal. Chem. 89, 1405 (2017)

8. J. Reguera, J. Langer, D.J. Aberasturi, L.M. LizMarzan, Anisotropic metal nanoparticles for surface enhanced Raman scattering. Chem. Soc. Rev. 46, 3866 (2017)

9. S. Wang, L.-L. Tay, H. Liu, A SERS and Electrical Sensor from Gas-Phase Generated Ag Nanoparticles Self-Assembled on Planar Substrates. Analyst 141, 1721-1733 (2016)

10. G. Lu, T.Z. Forbes, A.J. Haes, SERS detection of uranyl using functionalized gold nanostars promoted by nanoparticle shape and size. Analyst 141, 5137 (2016)

11. P.-J. Huang, L.-L. Tay, J. Tanha, S. Ryan, L.-K. Chau, SingleDomain Antibody-Conjugated Nanoaggregate-Embedded Beads for Targeted Detection of Pathogenic Bacteria. Chem. Eur. J. 15, 9330 (2009). https://doi.org/10.1002/chem.200901397

12. L.-L. Tay, P.-J. Huang, J. Tanha, S. Ryan, X. Wu, J. Hulse, L.-K. Chau, Silica encapsulated SERS nanoprobe conjugated to the bacteriophage tailspike protein for targeted detection of Salmonella. Chem. Commun. 48, 1024 (2012). https://doi.org/10.1039/C1CC1 $6325 \mathrm{~F}$

13. M. Moskovits, J. Yang, T. Haslett, SERS and the Single Molecule. In: Shalaev V.M. (eds) Optical Properties of Nanostructured Random Media. Topics in Applied Physics, vol 82. Springer, Berlin, Heidelberg (2002). https://doi.org/10.1007/3-540-44948-5_10

14. L.-L. Tay, J. Hulse, Surface-enhanced Raman and optical scattering in coupled plasmonic nanoclusters. J. Mod. Opt. 60, 1107 (2013)

15. L.-L. Tay, J. Hulse, D. Kennedy, J.P. Pezacki, Surface-Enhanced Raman and Resonant Rayleigh Scatterings From Adsorbate Saturated Nanoparticles. J. Phys. Chem. C 114, 7356 (2010). https:// doi.org/10.1021/jp9093222

16. J. Langer, D.J. de Aberasturi, J. Aizpurua et al., Present and Future of Surface-Enhanced Raman Scattering. ACS Nano 14, 28 (2020)

17. P.-J. Huang, L.-K. Chau, T.-S. Yang et al., Nanoaggregate-Embedded Beads as Novel Raman Labels for Biodetection. Adv. Funct. Mater. 19, 242 (2009). https://doi.org/10.1002/adfm.200800961

18. S. Milliken, J. Fraser, S. Poirier, J. Hulse, L.-L. Tay, Self-assembled vertically aligned Au nanorod arrays for surface-enhanced Raman scattering (SERS) detection of Cannabinol. Spectrochim. Acta A Mol. Biomol. 196, 222 (2018). https://doi.org/10.1016/j. saa.2018.01.030

19. O. Lyandres, N.C. Shah, C.R. Yonzon, J.T. Walsh, M.R. Glucksberg, R.P. Van Duyne, Real-Time Glucose Sensing by SurfaceEnhanced Raman Spectroscopy in Bovine Plasma Facilitated by a Mixed Decanethiol/Mercaptohexanol Partition Layer. Anal. Chem. 77, 6134 (2005)

20. L.-L. Tay, J. Hulse, Methodology for Binary Detection Analysis of Inkjet-Printed Optical Sensors for Chemical Detection. MRS Advances 6, 1 (2021). https://doi.org/10.1557/ s43580-021-00004-5

21. E.S. Ou, M. Hu, I. Naumov, A. Kim, W. Wu, A.M. Bratkovsky, X. Li, R.S. Williams, Z. Li, Nano Lett. 11, 2538 (2011) 